\title{
Coordination of voltage control in a power system operated by multiple transmission utilities
}

\author{
Yannick Phulpin \\ Supélec, France \\ yannick.phulpin@supelec.fr
}

\author{
Miroslav Begovic \\ Georgia Tech, USA \\ miroslav@ece.gatech.edu
}

\author{
Damien Ernst \\ University of Liège, Belgium \\ dernst@ulg.ac.be
}

\begin{abstract}
This paper addresses the problem of coordinating voltage control in a large-scale power system partitioned into control areas operated by independent utilities. Two types of coordination modes are considered to obtain settings for tap changers, generator voltages, and reactive power injections from compensation devices. First, it is supposed that a supervisor entity, with full knowledge and control of the system, makes decisions with respect to long-term settings of individual utilities. Second, the system is operated according to a decentralized coordination scheme that involves no information exchange between utilities. Those methods are compared with current practices on a 4141 bus system with 7 transmission system operators, where the generation dispatch and load demand models vary in discrete steps. Such a discretetime model is sufficient to model any event of relevance with respect to long-term system dynamics. Simulations show that centrally coordinated voltage control yields a significant improvement in terms of both operation costs and reserves for emergency control actions. This paper also emphasizes that, although it involves few changes with respect to current practices, the decentralized coordination scheme improves the operation of multi-utility power systems.
\end{abstract}

\section{Introduction}

Whereas active power imbalance leads to system wide frequency variations, voltage disturbance propagation is predominantly local. However, as emphasized in [1], those phenomena may propagate and subsequently turn into a system wide collapse. Postfestum analysis of numerous large-scale disturbances in different power systems have indeed reported that voltage instability phenomena are leading causes of blackouts [2]. To avoid voltage collapse, transmission system operators (TSOs) have implemented emergency control strategies to steer away the system from instability [3]. In addition, preventive voltage control schemes have been developed to maximize stability margins and minimize the operation costs. Many of the implemented voltage control schemes are hierarchical, i.e., they involve different control actions, objectives, and time/space delineations [4]. More specifically, fast control of reactive power injections from generators and flexible compensation devices is designed to undertake sudden changes in the system, resulting from the natural uncertainty among generation, demand, and transmission [5]. As those local actions rely on local measurements of voltage magnitudes and reactive power injections, the overall effectiveness of shortterm voltage control depends on the distribution of reactive power reserves. In practice, those reserves are scheduled by each TSO using a particular type of optimal power flow based on a steady-state forecast of operational conditions in its control area. As TSOs only have local knowledge and control of the system, coordination issues should be carefully addressed to avoid stressed conditions, especially close to interconnections [6], [7]. Consequently, two main trends in development of coordination strategies have emerged.

On one hand, centralized operation has been proposed to coordinate reactive power dispatch [8]. It usually consists of a centralized control center gathering information from different utilities, making decisions for the entire system, and advising system operators. Such a center was recently created to coordinate control actions between France and Belgium [9]. The main challenge for centralized coordination is the design of the decision-making process, which should have properties of fairness to be accepted by every party [10]. In addition, the necessary exchange of information might affect the robustness of the coordination scheme with respect to opportunistic behavior of the TSOs or the loss of communication channels, for example. 
To avoid those drawbacks, ad-hoc decentralized approaches have been proposed (see, e.g., [11]-[13]). In particular, Reference [14] presents a decentralized scheme for reactive power scheduling, where the TSOs concurrently schedule long-term voltage settings within their own control area, while representing the neighboring areas with external network equivalents whose parameters are fitted based on local measurements only. Previous papers [14], [15] have shown that such a coordination scheme could lead to close to optimal settings in the context of small-scale power system models. Furthermore, this promising scheme could be easily implemented on a real power system as it involves few departures from the current practices. Indeed, interconnected TSOs usually optimize their long-term voltage settings individually by representing the neighboring areas with other types of network equivalents based on practical operation recommendations [16].

This paper provides an evaluation of the centralized control scheme proposed in [8] and the decentralized control scheme proposed in [14] on a 4141 bus power system representing the western part of the UCTE system, which is operated by 7 different TSOs. Those strategies are also compared with the current practice in the UCTE system, modeled as a particular instance of the decentralized coordination scheme. It is the first time that these schemes are validated and compared with each other on such a large system. The evaluation relies on two indices that reflect both the ability of the scheme to lead to operation costs that could satisfy every TSO, and the potential impact of decentralized operation on fast reactive power compensation and voltage control.

The paper is organized as follows. In Section 2, the problem of reactive power scheduling in a multiTSO system is formalized. In Section 3, the centralized optimization scheme is described, while the decentralized coordination scheme is detailed in Section 4. The benchmark system, evaluation indices, and simulation results are presented in Section 5. Finally, Section 6 concludes and discusses some future research directions.

\section{Problem formulation}

This section details the problem of reactive power scheduling in a multi-TSO power system. In the first part of this section, the single-area reactive power dispatch problem is formalized. Then, the multi-TSO problem is described.

\subsection{Single area problem}

Reactive power scheduling is usually carried out every ten minutes to one hour [17]. It consists of the optimization at each discrete time instant $k$ of a power system characterized by operational conditions $r(k)$, which represent the scheduled load demand, active power generation pattern, and network topology at instant $k$.

In this context, the control variables are the tap settings, generators' voltages, and compensation devices reactive power injections [18] that will be applied at instant $k$. Usually, they are represented by a vector ${ }^{1}$ u. In a real power system, reactive power settings can change between two discrete instants. However, as their variations are relatively minor compared to the magnitude of compensation, they can be neglected and approximated with constant values without any major loss of accuracy or concern.

The reactive power scheduling process is depicted in Figure 1. It assumes that every TSO perfectly predicts at the instant $k-1$ the operating conditions, under which its control area will be operated at the instant $k$. We will suppose in this paper that such a forecast is available.

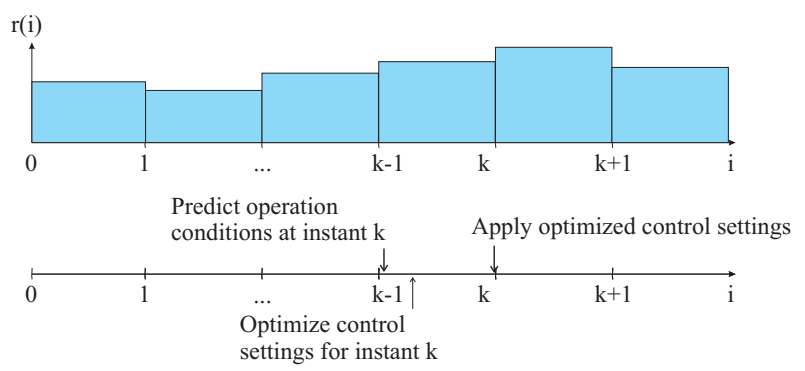

Figure 1. Chronology of control actions for reactive power scheduling in a time-varying power system.

We characterize the optimal power flow problem faced by a TSO at instant $k-1$ as follows ${ }^{2}$ :

$$
\min _{\mathbf{u}} C^{k}(\mathbf{u})
$$

subject to

$$
\begin{aligned}
& \mathbf{f}^{k}(\mathbf{u})=\mathbf{0} \\
& \mathbf{g}^{k}(\mathbf{u}) \leq \mathbf{0}
\end{aligned}
$$

where $C^{k}(\mathbf{u}), \mathbf{f}^{k}(\mathbf{u})$, and $\mathbf{g}^{k}(\mathbf{u})$ represent the objective

1. Bold fonts are used in this paper to highlight multi-dimensional variables and functions.

2. Such a characterization is common in the power system literature (see, e.g., [19], [20]). 
function $^{3}$ and the equality and inequality constraint functions, respectively.

The formulation of the objective function $C^{k}(\mathbf{u})$ is particularly important for TSOs and many research papers have focused on this problem [21]-[23]. While implicit objectives are mainly concerned with the operational costs and voltage stability, those can be explicitly formulated through different criteria, many of them being reported in [24]. In this paper, we consider that TSOs focus on either active power losses or voltage profile.

In practice, the equality constraint (2) represents the power flow equations and the distributed loss compensation (distributed slack bus), while the inequality constraint (3) represents various physical limits that need to be taken into account when operating a power system (e.g., bus voltage limits, transmission line current limits, minimum and maximum reactive power injections).

\subsection{Multi-TSO problem}

In this paper, the power system under consideration has $N$ control areas $1,2, \ldots, N$ operated by $T S O_{1}$, $\mathrm{TSO}_{2}, \ldots$, and $T S \mathrm{~T}_{N}$, respectively. Because the dynamics of interconnected areas of an AC system are coupled, the objective and constraint functions $C_{i}^{k}$, $\mathbf{f}_{i}^{k}$, and $\mathbf{g}_{i}^{k}$ of every $T S O_{i}$ should be expressed as functions of the control variable $\mathbf{u}$ that appends the vectors of individual control settings $\mathbf{u}_{1}, \mathbf{u}_{2}, \ldots, \mathbf{u}_{N}$ for each TSO. Let us denote $\mathbf{f}^{k}(\mathbf{u})$, and $\mathbf{g}^{k}(\mathbf{u})$ the concatenation of the $N$ respective constraint functions, and $\mathbf{U}^{\mathbf{k}}$ the set of control actions $\mathbf{u}$ such that $\mathbf{f}^{k}(\mathbf{u})=\mathbf{0}$ and $\mathbf{g}^{k}(\mathbf{u}) \leq \mathbf{0}$.

2.2.1. Constraint violations. When a $T S O_{i}$ is unaware of the operating conditions in the other control areas, it has to formulate its own objective function $\hat{C}_{i}^{k}\left(\mathbf{u}_{i}\right)$, and constraint functions $\hat{\mathbf{f}}_{i}^{k}\left(\mathbf{u}_{i}\right)$ and $\hat{\mathbf{g}}_{i}^{k}\left(\mathbf{u}_{i}\right)$ as functions of its internal control variable $\mathbf{u}_{i}$ only ${ }^{4}$. In this case, there may exist particular settings $\mathbf{u}^{*}$ such that $\hat{\mathbf{f}}_{i}^{k}\left(\mathbf{u}_{i}^{*}\right)=0$ and $\hat{\mathbf{g}}_{i}^{k}\left(\mathbf{u}_{i}^{*}\right) \leq \mathbf{0}, \forall i \in\{1, \ldots, N\}$ but $\mathbf{u}^{*} \notin \mathbf{U}^{\mathbf{k}}$.

If such a case were to occur in a real system, shortterm voltage control would then modify the control settings of generators and fast reacting compensation devices [5] to obtain new settings $\mathbf{u}^{S}$ that comply

3. The superscript ${ }^{k}$ denotes the fact that the associated function depends on $r(k)$.

4. The symbol ${ }^{\wedge}$ on $C_{i}^{k}, \mathbf{f}_{i}^{k}$, and $\mathbf{g}_{i}^{k}$ specifies that, since a $T S O_{i}$ does not know the system topology, generation pattern, load demand, and control actions in the other areas, it can only formulate its own objective and constraints as functions of its own system state determined by $\mathbf{u}_{i}$. with the system's constraints. We will suppose later in our simulations that these settings correspond to the solution of the following optimization problem:

$$
\mathbf{u}^{S}=\underset{\mathbf{u}}{\arg \min }\left\|\mathbf{u}-\mathbf{u}^{*}\right\|
$$

subject to

$$
\begin{array}{r}
\mathbf{h}^{k}\left(\mathbf{u}, \mathbf{u}^{*}\right)=\mathbf{0}, \\
\mathbf{u} \in \mathbf{U}^{\mathbf{k}},
\end{array}
$$

where Equation (5) is to keep settings for tap changers and slow compensation devices unchanged.

Such a procedure should be avoided, since it tends to reduce reactive power reserves that might be required to counteract fast voltage variations. Consequently, constraint violations in reactive power scheduling are correlated with higher system vulnerability with respect to sudden disturbances.

2.2.2. Objective of the coordination. In the context of a large-scale power system operated by multiple TSOs with individual (possibly conflicting) objectives, coordination should lead at any instant $k$ to control settings $\mathbf{u}$ such that:

- Constraint violations require as little fast voltage control action as possible.

- The individual cost $C_{i}^{k}(\mathbf{u})$ supported by every $T S O_{i}$ is as close as possible to its minimum.

In addition, it is expected that the coordination involves as little information exchange as possible to reduce the system vulnerability with respect to opportunistic behavior of some utilities, or loss of a communication channel, for example.

\section{Centralized optimization}

We suppose that a centralized control center, with full knowledge and control of the system, requires that each TSO adjusts the settings of generators, compensation devices, and tap changers within its control area. Hence, the centralized control center has to solve the following multi-party optimization problem:

$$
\min _{\mathbf{u} \in \mathbf{U}^{\mathbf{k}}}\left[C_{1}^{k}(\mathbf{u}), C_{2}^{k}(\mathbf{u}), \ldots, C_{N}^{k}(\mathbf{u})\right]
$$

While many approaches in multi-objective optimization could be applied to solve this problem [25], we assume that the centralized control center uses a socalled "compromise method," which elects the solution $\mathbf{u}^{*}$ whose cost vector $\left[C_{1}^{k}\left(\mathbf{u}^{*}\right), C_{2}^{k}\left(\mathbf{u}^{*}\right), \ldots, C_{N}^{k}\left(\mathbf{u}^{*}\right)\right]$ is the closest to the "Utopia point" $\mathbf{C}_{u t}^{k}$ defined by

$$
\mathbf{C}_{u t}^{k}=\left[C_{1}^{k}\left(\mathbf{u}^{k^{1 *}}\right), C_{2}^{k}\left(\mathbf{u}^{k^{2 *}}\right), \ldots, C_{N}^{k}\left(\mathbf{u}^{k^{N *}}\right)\right]
$$


where $\mathbf{u}^{k^{i *}}$ is the solution of

$$
\mathbf{u}^{k^{i *}}=\underset{\mathbf{u} \in \mathbf{U}^{\mathbf{k}}}{\arg \min } C_{i}^{k}(\mathbf{u}) .
$$

The distance between two cost vectors is chosen as the Euclidean distance between normalized costs. The normalization factors are chosen as in [8] to ensure that the solution of Problem (7) has some properties of fairness. More specifically, for a particular cost function $C_{i}^{k}(\mathbf{u})$, the normalization factor is defined as the product of the two terms ${ }^{5} C_{i}^{k^{\circ}}$ and $\chi_{i}^{k}$, and the normalized cost function $\bar{C}_{i}^{k}(\mathbf{u})$ is computed using the following equation:

$$
\overline{C_{i}^{k}}(\mathbf{u})=\frac{C_{i}^{k}(\mathbf{u})}{C_{i}^{k^{\circ} \times \chi_{i}^{k}}},
$$

where

$$
C_{i}^{k^{\circ}}=\sum_{j=1}^{N} \frac{C_{i}^{k}\left(\mathbf{u}^{k^{j *}}\right)-C_{i}^{k}\left(\mathbf{u}^{k^{i *}}\right)}{N},
$$

and

$$
\chi_{i}^{k}=\sum_{j=1}^{N} \frac{C_{j}^{k}\left(\mathbf{u}^{k^{i *}}\right)-C_{j}^{k}\left(\mathbf{u}^{k^{j *}}\right)}{C_{j}^{k^{\circ}}} .
$$

Hence, the optimization problem to be solved by the centralized control center can be formulated as follows:

$$
\mathbf{u}^{*}=\underset{\mathbf{u} \in \mathbf{U}^{\mathbf{k}}}{\arg \min } \sum_{i=1}^{N}\left[\overline{C_{i}^{k}}(\mathbf{u})-\overline{C_{i}^{k}}\left(\mathbf{u}^{k^{i *}}\right)\right]^{2} .
$$

It can be easily shown [25] that such an optimization scheme leads systematically to a solution that is Paretooptimal, i.e., for which there exists no other solution in $\mathrm{U}^{\mathrm{k}}$ that improves at least one objective without making any other objective worse.

\section{Decentralized operation}

While the centralized optimization scheme detailed in the previous section leads systematically to a Paretooptimal solution that does not violate the constraints, it requires all TSOs to report sensitive information to a centralized control center and surrender to this center some authority over their control actions. Additionally, the scheme might also not be robust with respect to the biased behavior of one or several parties [8]. To avoid these shortcomings, we propose to use a decentralized coordination scheme with no need of information exchange, where every TSO maintains its prerogatives (objective function, control actions, load forecasting, etc).

5. $C_{i}^{k^{\circ}}$ and $\chi_{i}^{k}$ can not be equal to zero as long as there exists a single different solution for each Problem (9), which is the case for the simulations reported in this paper.
The main features of the decentralized control scheme are introduced in [15]. In such a decentralized scheme, every TSO computes the new values of its control actions to be applied at instant $k$ along a specific procedure. For each TSO, this procedure consists in using the record of some measurements at its interconnections to fit some simple external network models, and then solving in a greedy way its own power scheduling problem.

\subsection{Improved coordination}

While different types of network equivalents are possible, reference [14] shows that modeling the external network with a constant active and reactive power injection at every point of interconnection yields close to optimal performance even if the TSOs' control actions are not coordinated by a centralized authority.

The equivalents used by every $T S O_{i}$ are characterized by a parameter $\mathbf{z}_{i}^{*}(k)$, which gathers all values of active and reactive power parameters of the equivalents used by $T S O_{i}$ to represent the external system. The parameter $\mathbf{z}_{i}^{*}(k)$ is computed using an exponential recursive least squares technique based on a record of measurements at the previous instants $\mathbf{z}_{i}^{S}(j), \forall j \in\{1, \ldots, k-1\}$. More specifically, $\mathbf{z}_{i}{ }^{*}(k)$ is determined by solving the following minimization problem:

$$
\min _{\mathbf{z}_{i}} \sum_{j=0}^{k-1} \beta^{1+j-k} \times\left\|\mathbf{z}_{i}^{S}(j)-\mathbf{z}_{i}\right\|^{2},
$$

where $\beta$ represents the memory factor for the measurements.

At instant $k-1$, every $T S O_{i}$ thus solves the following optimization problem:

$$
\min _{\mathbf{u}_{i}} \hat{C}_{i}^{k}\left(\mathbf{u}_{i}\right),
$$

under the inequality and equality constraints

$$
\begin{aligned}
& \hat{\mathbf{f}}_{i}^{k}\left(\mathbf{u}_{i}\right)=\mathbf{0}, \\
& \hat{\mathbf{g}}_{i}^{k}\left(\mathbf{u}_{i}\right) \leq \mathbf{0},
\end{aligned}
$$

where the equality Constraint (17) depends now on the value of $\mathbf{z}_{i}^{*}(k)$, since it represents also the constraints imposed by the external network equivalents used by $T S O_{i}$ at instant $k$.

The solutions $\mathbf{u}_{1}^{*}(k), \mathbf{u}_{2}^{*}(k), \ldots, \mathbf{u}_{N}^{*}(k)$ are appended onto $\mathbf{u}^{*}(k)$, which is applied to the interconnected system at instant $k$. As introduced in Section 2.2.1, if $\mathbf{u}^{*}(k)$ does not comply with Constraints (2) and (3), faster voltage control loops will change the operation settings and lead to $\mathbf{u}^{S}(k) \in \mathbf{U}^{\mathbf{k}}$, solution of 
Problem (4) under Constraints (5)-(6). Active power and reactive power flows at each interconnection, represented by $\mathbf{z}_{i}^{S}(k)$, are then measured by each TSO, which updates its record of measurements at the interconnections.

\subsection{Utility practice}

The decentralized control scheme introduced in [15] is related to current practices in interconnected systems. Indeed, many transmission system operators perform their reactive power scheduling periodically by modeling the external system with simple electrical equivalents. This approach could raise problems, for example, when neighboring utilities are maintaining different nominal voltages in their networks on the two sides of an interconnection (e.g., 400 and $390 \mathrm{kV}$ ). Interconnected TSOs have thus developed operation practices to account for such conditions and avoid violations of the interface constraints. For example, the UCTE system recommends in its operation handbook [16] that interconnected TSOs should coordinate their actions and agree on an acceptable voltage range at each interconnection, which can be roughly formulated as a zero reactive power flow at every interconnection.

Based on this UCTE recommendation, we will therefore assume that a current practice for decentralized operation of multi-area power systems is approximately equivalent to a particular instance of the scheme proposed in [15], where TSOs assume reactive power injections at the interconnections equal to zero. Besides, we will assume that the active power injections at the interconnections are set using the procedure detailed in the previous subsection. With such a procedure, the TSOs will set the active power injection at each interconnections to a weighted average of its previous values. The rationale behind this assumption is that TSOs usually do not want to interfere with the active power exchanges at the interconnections when optimizing their voltage control settings.

\section{Evaluation of the coordination schemes}

While it can be easily demonstrated that centralized optimization systematically leads to a Pareto-optimal solution, the ability of decentralized coordination to lead to nearly optimal solutions is difficult to prove.

To assess the performance of the decentralized coordination schemes, we will therefore rely on simulation results. Those have been generated for a 4141 bus system with 7 TSOs with many different operational conditions. Both this system and some specificities of the control schemes are described in Section 5.1. The evaluation is based on two evaluation indexes, presented in Section 5.2. Finally, results are presented and commented in Section 5.3.

\subsection{Benchmark system}

The evaluation is carried out on a reduced system representing the western part of the UCTE system (including Spain, France, Belgium, The Netherlands, Germany, Switzerland, and Italy). It is composed of 4141 buses, 624 generators, 6419 branches, and 7 TSOs.

The generation and demand patterns are set on a hourly basis depending on four snapshots of the UCTE system on December 17, 2007 at 3.30 a.m., 10.30 a.m., 12.30 p.m., and 7.30 p.m. In addition, a scaling factor is applied to load demand in every control area according to real hourly measurements reported on the ENTSOE (European network of transmission system operators for electricity) website [26]. We report simulation results corresponding to 744 hours (i.e., one month), with one discrete instant per hour. Although the snapshots correspond to a day of heavy load for the UCTE system, a variety of operational conditions is considered with scaling factors of each area varying from 0.55 through 1.31 .

The optimization variable $\mathbf{u}$ represents the settings for tap changers and voltage magnitudes for the generators and compensation devices in the reduced system. The objectives of the TSOs were chosen among the common practices introduced in Section 2.1. More specifically, the objective of each $T S O_{i}$ is defined as the minimization of either active power losses $C_{L}$ or the optimization of a voltage profile with respect to a voltage magnitude reference value $V_{\text {ref }}$. This latter type of objective function, referred to by $C_{V_{\text {ref }}}$, is defined as follows:

$$
C_{V_{\text {ref }}}(\mathbf{u})=\sqrt{\sum_{i \in\left\{1, \ldots, N_{B}\right\}}\left(V_{B_{i}}(\mathbf{u})-V_{\text {ref }}\right)^{2}},
$$

where $N_{B}$ represents the number of buses in the control area and $V_{B_{i}}(\mathbf{u})$ is the voltage magnitude at bus $i$, in per unit. The type of objective of each TSO is arbitrarily chosen as detailed in Table 1.

The value of the memory factor $\beta$ used by the decentralized coordination schemes is chosen equal to 0.5 . 
Table 1. Objective function of every $T S O_{i}$ in the benchmark system.

\begin{tabular}{|c|c|}
\hline$i$ & $C_{i}$ \\
\hline \hline 1 and 2 & $C_{V_{\text {ref }}}\left(V_{\text {ref }}=0.97\right.$ p.u. $)$ \\
\hline 3,4 , and 5 & $C_{V_{\text {ref }}}\left(V_{\text {ref }}=1.01\right.$ p.u. $)$ \\
\hline 6 and 7 & $C_{L}$ \\
\hline
\end{tabular}

\subsection{Evaluation indices}

As emphasized in Section 2.2.2, the performance of a coordination scheme depends on two criteria.

First, a decentralized coordination scheme may lead at instant $k$ to a control setting $\mathbf{u}^{*}(k)$ that does not necessarily belong to $U^{k}$. As discussed earlier in the paper, we assume in such cases that faster controllers will modify the control variables of the system to alleviate the constraint violations. The efforts made by these faster controllers will be used to define an evaluation index, which will be referred to as "effort index." More specifically, the effort index $E(k)$ is defined as the Euclidean distance between $\mathbf{u}^{*}(k)$ and $\mathbf{u}^{S}(k)$, as follows:

$$
E(k)=\left\|\mathbf{u}^{S}(k)-\mathbf{u}^{*}(k)\right\| .
$$

Note that since the difference between $\mathbf{u}^{*}(k)$ and $\mathbf{u}^{S}(k)$ concerns only voltage settings that are expressed in per unit, we can consider that a value of index $E(k)$ under 0.001 represents no significant constraint violation. On the contrary, a value of $E(k)$ over 0.1 means that at least one of the voltage settings had to be modified significantly. As emphasized in Section 2.2.1, this implies a change in reactive power reserves available for fast voltage control actions, which reduces the system ability to deal with critical situations.

Second, a coordination scheme is considered efficient, if it constantly leads at every instant $k$ to control setting $\mathbf{u}^{S}(k)$, whose associated costs are minimal for every $T S O_{i}$. Based on this, we have chosen to relate the efficiency evaluation to the distance $D(k)$ that is minimized for centralized optimization in Equation (13). The evaluation relies thus on a sub-optimality index $D(k)$ assessed as follows:

$$
D(k)=\sqrt{\sum_{i=1}^{N}\left[\overline{C_{i}^{k}}\left(\mathbf{u}^{S}(k)\right)-\overline{C_{i}^{k}}\left(\mathbf{u}^{i *}(k)\right)\right]^{2}} .
$$

As the evaluation index $D(k)$ relies on normalized cost functions, it can not be directly related to a physical or economic metrics. However, it allows to equally consider objectives that have different natures, as it is the case for the benchmark system. It must be noted that the centralized optimization scheme introduced in Section 3 leads to the lowest sub-optimality that can be reached. On the contrary, a setting for which $D(k)$ is around 1 corresponds for at least one TSO to operation costs significantly higher than what it could expect. A coordination scheme leading to a value of $D(k)$ of the order of 1 is thus unlikely to satisfy every party.

\subsection{Simulation results}

Figure 2 presents the evolution of the sub-optimality index $D(k)$ for centralized optimization, decentralized coordination, and current practice. We see that the decentralized control scheme used with constant power injection equivalents where the active and reactive power injections are fitted to past observations performs better than the current practice - modeled as a decentralized power scheme where the reactive power injection is chosen always equal to zero and the active power injection is fitted to past observations - since it tends to lead to lower values of $D(k)$. More specifically, the average distance to the "Utopia point" is equal to 0.47 with the improved decentralized coordination and 0.53 with the current practice. This result suggests therefore that part of the sub-optimality related to the lack of coordination could be avoided by a small change in operational practices. However, this improvement in performance is limited as it leads systematically to operation settings corresponding to individual costs that are significantly higher than what every party could expect with a centralized coordination. Indeed, the average value of index $D(k)$ it is equal to 0.12 with the centralized optimization scheme.

Figure 3 depicts the evolution of the short-term voltage control effort $E(k)$ that is necessary to maintain the system within its operation limits after application of the settings assessed by a decentralized control scheme. It shows that fitting both the active and reactive power injections of the equivalents to the past observations leads to a smaller effort ( 0.22 in average) than with the current practice ( 0.29 in average). Note that those values of the effort index reveal that a significant effort of faster voltage control is required to keep the system within its limits, which in turn can challenge the realtime operation of the interconnected system.

\section{Conclusion}

This paper addresses the problem of coordinating voltage control in a large-scale power system operated by several TSOs, where the load demand evolves according to a discrete-time load curve. It provides a comparison of three control schemes in the context 


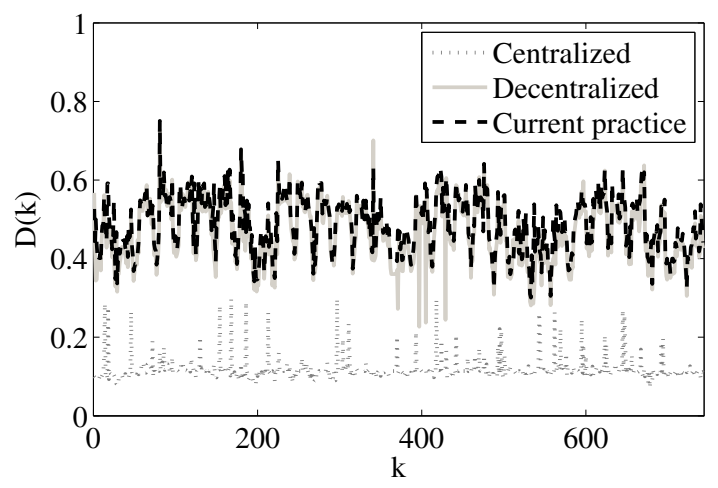

Figure 2. Evolution of the performance index $D(k)$ with the 4141 bus benchmark system under timevarying operational conditions. A discrete instant $k$ corresponds to one hour.

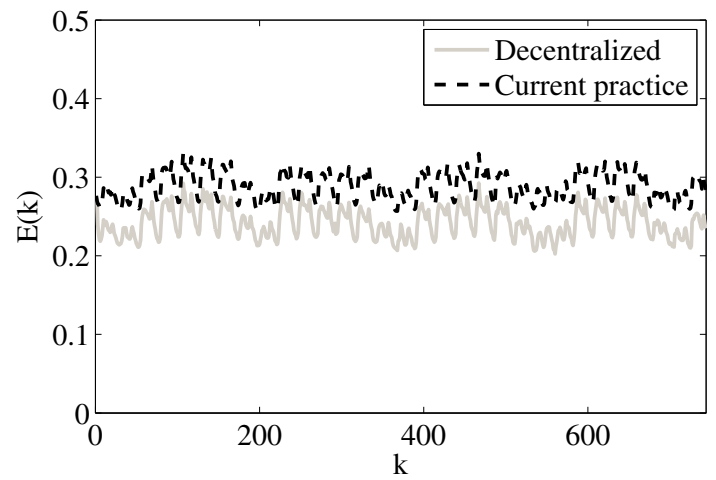

Figure 3. Evolution of the effort index $E(k)$ with the 4141 bus benchmark system under time-varying operational conditions. A discrete instant $k$ corresponds to one hour.

of a 4141 bus system with 7 TSOs, where both the ability of the coordination scheme to satisfy every TSO and the effects of solutions that do not comply with the constraints on fast voltage control reserves are analyzed.

Unlike results presented in previous papers, which focused on some smaller scale models without tap changers, simulation results show that decentralized operation can induce a significant solicitation of voltage control reserves. They also show that a current practice in multi-TSO voltage control - formalized in this paper as a particular instance of a decentralized optimization scheme - could be improved through slight modifications in scheduling reactive power exchanges at the interconnections. In addition, the new results highlight that decentralized coordination leads to individual operation costs that are significantly higher than those that would occur with a centralized control scheme. The numerous simulations presented in this paper are based on only four snapshots of a real system under heavy load conditions. Therefore, we do not claim that our conclusions are universally valid. In particular, the shortcomings of decentralized operation may not be a concern, when the system faces a lower load demand.

Nevertheless, those observations emphasize the potential benefits of centralized coordination for voltage control, for which TSOs must agree on a decisionmaking scheme. To this extent, several issues related to the large-scale application of the centralized control scheme remain to be addressed. In particular, more advanced computational techniques should be investigated to identify optimal settings when an objective is almost independent of some control variables (e.g., the voltage setting for a generator located far away from the area under consideration). Further research should also focus on the robustness of the centralized coordination scheme with respect to the loss of some communication channels, among others.

\section{Acknowledgment}

Damien Ernst is a Research Associate of the Belgian FNRS, from which he acknowledges the financial support.

\section{References}

[1] T. Van Cutsem and C. Vournas, Voltage Stability of Electric Power Systems. Kluwer Academic Publishers, 1998.

[2] C. Taylor, Power System Voltage, Stability. New York: McGraw Hill, 1994.

[3] S. Greene, I. Dobson, and F. Alvarado, "Sensitivity of the loading margin to voltage collapse with respect to arbitrary parameters," IEEE Transactions on Power Systems, vol. 12, pp. 262-268, February 1997.

[4] Y. Rebours, D. Kirschen, M. Trotignon, and S. Rossignol, "A survey of frequency and voltage control ancillary services - part I: technical features," IEEE Transactions on Power Systems, vol. 22, pp. 350-357, February 2007.

[5] C. Taylor, "Reactive power today, best practices to prevent blackouts," IEEE Power and Energy Magazine, vol. 4, pp. 104-102, September 2006.

[6] J. Bialek, "Are blackouts contagious?," IEE Power Engineer, vol. 17, pp. 10-13, December 2003. 
[7] UCTE, "Final Report - system disturbance on 4 November 2006," tech. rep., UCTE, January 2007.

[8] Y. Phulpin, M. Begovic, M. Petit, and D. Ernst, "A fair method for centralized optimization of multiTSO power systems," International Journal of Electric Power and Energy Systems, vol. 31, pp. 482-488, September 2009.

[9] Coreso, "http://www.coreso.eu."

[10] J. Buchanan, "The relevance of pareto optimality," Journal of conflict resolution, vol. 6, pp. 341-354, december 1962.

[11] A. Conejo and J. Aguado, "Multi-area coordinated decentralized DC optimal power flow," IEEE Transactions on Power Systems, vol. 13, pp. 1272-1278, November 1998.

[12] A. Marinakis, M. Glavic, and T. Van Cutsem, "Control of phase shifting transformers by multiple transmission system operators," in Proc. of the Power Tech 2007, (Lausanne, Switzerland), July 2007.

[13] G. Hug-Glanzmann and G. Andersson, "Decentralized optimal power flow control for overlapping areas in power systems," IEEE Transactions on Power Systems, vol. 24, pp. 327-336, February 2009.

[14] Y. Phulpin, M. Begovic, M. Petit, J. Heyberger, and D. Ernst, "Evaluation of network equivalents for voltage optimization in multi-area power systems," IEEE Transactions on Power Systems, vol. 24, pp. 729-743, May 2009.

[15] Y. Phulpin, M. Begovic, M. Petit, and D. Ernst, "Decentralized reactive power dispatch for a time-varying multi-TSO system," in Proc. of the HICSS 2009, (Hawaii, USA), pp. 1 - 8, January 2009.

[16] UCTE, "UCTE Operation Handbook," 2009.

[17] N. Martins, N. Macedo, L. Lima, and H. Pinto, "Control strategies for multiple static VAr compensators in long distance voltage supported transmission systems," IEEE Transactions on Power Systems, vol. 8, pp. 1107-1117, August 1993.

[18] P. Kundur, Power System Stability and Control. McGraw-Hill, 1994.

[19] H. Dommel and W. Tinney, "Optimal power flow solutions," IEEE Transactions on Power Apparatus and Systems, vol. PAS-87, no. 10, pp. 1866-1876, 1968.

[20] F. Capitanescu, M. Glavic, D. Ernst, and L. Wehenkel, "Interior-point based algorithms for the solution of optimal power flow problems," Electric Power Systems Research, vol. 77, pp. 508-517, April 2007.

[21] F. Graf, "Real-time application of an optimal power flow algorithm for reactive power allocation of the RWE energy control center," in Proc. of the IEE Colloquium on International Practices in Reactive Power Control, (London, UK), pp. 71-74, April 1993.
[22] T. Menezes, T. Da Silva, C. Affonso, and V. Da Costa, "MVAr management on the pre-dispatch problem for improving voltage stability margin," IEE Proc. on Generation Transmission and Distribution, vol. 151, pp. 665-672, November 2004.

[23] D. Thukaram, L. Jenkins, and K. Visakha, "Optimum allocation of reactive power for voltage stability improvement in AC-DC power systems," IEE Proc. on Generation Transmission and Distribution, vol. 153, pp. 237-246, March 2006.

[24] R. Salgado and M. Irving, "Framework for the analysis of reactive power dispatch in energy pools," IEE Proc. on Generation, Transmission, Distribution, vol. 151, pp. 167-174, March 2004.

[25] R. Marler and J. Arora, "Survey of multi-objective optimization methods for engineering," Structural and Multidisciplinary Optimization, vol. 26, no. 6, pp. 369395, 2004.

[26] European Transmission System Operators, "System vertical load data." Available at : http://www.etsovsita.org, 2008. 